\title{
Foreword to the Special Issue on Hyperspectral Remote Sensing and Imaging Spectroscopy
}

$\mathbf{H}$ YPERSPECTRAL remote sensing has emerged as a powerful tool to understand phenomena at local and global scales by virtue of imaging through a diverse range of platforms, including terrestrial in-situ imaging platforms, unmanned and manned aerial vehicles, and satellite platforms. By virtue of imaging over a wide range of spectral wavelengths, it is possible to characterize object specific properties very accurately. As a result, hyperspectral imaging (also known as imaging spectroscopy) has gained popularity for a wide variety of applications, including environment monitoring, precision agriculture, mineralogy, forestry, urban planning, and defense applications.

The increased analysis capability comes at a cost - there are a variety of challenges that must be overcome for robust image analysis of such data, including high dimensionality, limited sample size for training supervised models, noise and atmospheric affects, mixed pixels, etc. This special issue (SI) presents 26 papers that represent some of the recent developments in image analysis algorithms and unique applications of hyperspectral imaging data. Specifically, this SI represents the following broad topics.

1) Contemporary and emerging machine learning architectures for image analysis.

2) Advances in spectral unmixing for image analysis.

3) Real-time compression and compressive sensing.

4) Denoising.

5) Applications leveraging the information provided by hyperspectral earth observations.

In Wang et al. a group low-rank nonnegative matrix factorization approach is proposed for spectral unmixing. In Zhang et al. image fusion of multispectral and hyperspectral imagery is undertaken via a spatial-spectral graph-regularized low-rank tensor decomposition. Al-Suwaidi et al. propose feature ensemble based novelty detection for analysis of plant hyperspectral datasets. Matteoli et al. present a target recognition approach within anomalous regions of interest in hyperspectral images. In Du et al. a low-rank matrix factorization based approach is paired with a band-specific noise model for hyperspectral denoising. In Zhang et al. cascaded random forests are proposed for hyperspectral image classification. In Yu et al. a mixed pixel hyperspectral classification approach is developed and presented. In Gan et al. a weighted kernel sparse representation model is developed for hyperspectral classification. Wu et al. present a GPU parallel implementation for hyperspectral image classification that utilizes spatial information. Liu et al. undertake hyperspectral classification via least-square support vector machines. Bascones et al. present an FPGA implementation for real-time hyperspectral lossless compression. Kang et al. present a Gabor filtering based deep network for hyperspectral classification. Tang et al. present an approach that integrates spatial information with the normalized P-linear algorithm for spectral unmixing. Salehani et al. present a sparse hyperspectral unmixing algorithm. Mukherjee et al. present a spatially constrained angular subspace learning approach for hyperspectral image classification. Liao et al. present a constrained manifold learning approach tailored to hyperspectral imagery visualization. Wang et al. present an approach to hyperspectral image restoration based on total variation regularized low-rank tensor decomposition. Chakravortty et al. present a subpixel timeseries hyperspectral analysis approach for dynamic mangrove eco-system monitoring. Deborah et al. present assessment protocols and comparison of ordering relations for spectral image processing. Wang et al. propose an unmixing based approach to compressed sensing reconstruction. Chang et al. present a review of works related the virtual dimensionality of hyperspectral data. Zaouali et al. integrate three-dimensional (3-D) shearlet transforms with Joint Sparse Representation for hyperspectral classification. Zhang et al. estimate Chlorophyll-a in turbid waters using SAR and hyperspectral imagery over Lake Taihu. Sellami et al. present an approach to semantic interpretation of hyperspectral imagery. Pieper et al. present the effects of wavelength calibration mismatch on temperatureemissivity separation techniques. Li et al. present an approach to use hyperspectral imagery for effective mineral mapping via multiparameter optimization.

\author{
S. PRASAD, Guest Editor \\ University of Houston \\ Houston, TX 77004 USA
}

W. LIAO, Guest Editor
Ghent University
Ghent 9000, Belgium

M. HE, Guest Editor

Northwestern Polytechnical University

Xi' an 710072 , China

J. ChAnussot, Guest Editor

University Grenoble Alpes, CNRS,

Grenoble INP, GIPSA-lab

Grenoble 38400, France

Digital Object Identifier 10.1109/JSTARS.2018.2820938

1939-1404 @ 2018 IEEE. Personal use is permitted, but republication/redistribution requires IEEE permission. See http://www.ieee.org/publications_standards/publications/rights/index.html for more information. 


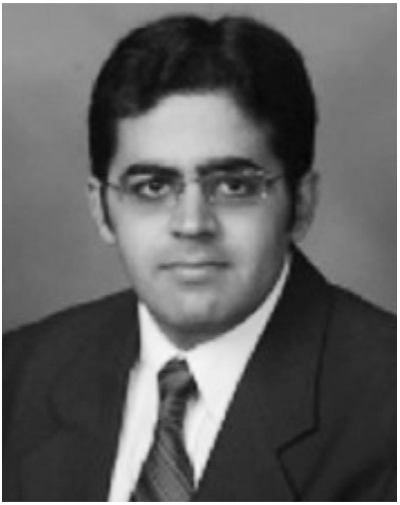

Saurabh Prasad (S'05-M'09-SM'14) received the B.S. degree from Jamia Millia Islamia, New Delhi, India, in 2003, the M.S. degree from Old Dominion University, Norfolk, VA, USA, in 2005, and the Ph.D. degree from Mississippi State University, Starkville, MS, USA, in 2008, all in electrical engineering.

He is currently an Assistant Professor with the Electrical and Computer Engineering Department, University of Houston (UH), Houston, TX, USA, where he leads a research group on image processing and machine learning. His research interests include statistical pattern recognition, adaptive signal processing, compressive sensing, and theoretical foundations of machine learning with applications to remote sensing and biomedical applications. In particular, his current research work involves design of optimal sparse representation frameworks, active learning, Bayesian inference, kernel machines, and deep learning for robust image analysis to address issues related to small-training-sample-size, mixed pixels, low SNR, and varying illumination.

Dr. Prasad was a recipient of two research excellence awards (2007 and 2008) during his Ph.D. study at Mississippi State University, including the university wide outstanding graduate student research award. In July 2008, he was also a recipient of the Best Student Paper Award at the IEEE International Geoscience and Remote Sensing Symposium in 2008 held in Boston, MA, the State Pride Faculty Award at Mississippi State University for his academic and research contributions in October 2010, the NASA New Investigator (Early Career) Award in 2014, and the Junior Faculty Research Award at UH, in 2017.

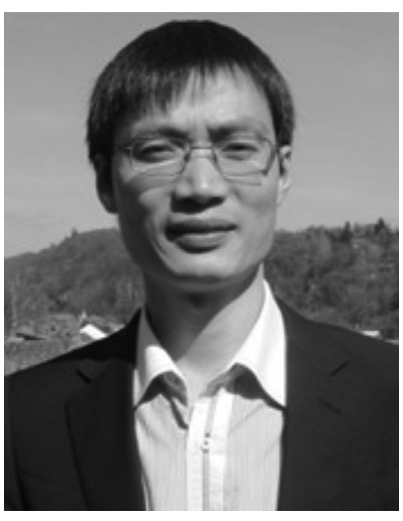

Wenzhi Liao (S'10-M'14-SM'16) received the B.S. degree in mathematics from Hainan Normal University, Haikou, China, in 2006, the Ph.D. degree in engineering from South China University of Technology, Guangzhou, China, in 2012, and the Ph.D. degree in computer science engineering from Ghent University, Ghent, Belgium, in 2012.

Since 2012, he has been working as a Postdoctoral with Ghent University and then as a Postdoctoral Research Fellow with Research Foundation Flander. His current research interests include pattern recognition, remote sensing, and image processing. In particular, his interests include mathematical morphology, multitask feature learning, multisensor data fusion, and hyperspectral image restoration.

Dr. Liao is a member of the Geoscience and Remote Sensing Society (GRSS) and IEEE GRSS Data Fusion Technical Committee. He was a recipient of the "Best Paper Challenge" Awards on both 2013 IEEE GRSS Data Fusion Contest and the 2014 IEEE GRSS Data Fusion Contest.

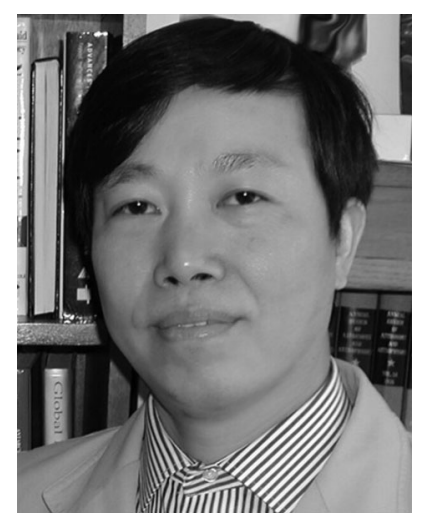

Mingyi He (M'06-SM'16) received the B.Eng. and M.S. degrees in electronic engineering and signal processing from Northwestern Polytechnical University (NPU), Xi'an, China, in 1982 and 1985, respectively, and the Ph.D. degree in signal and information processing from Xidian University, Xi'an, China, in 1994.

Since 1985, he has been with the School of Electronics and Information, NPU, where he has been a Full Professor since 1996. He is the Founder and Director of Shaanxi Key Laboratory and International Research Center for Information Acquisition and Processing, and the Director and Chief Scientist of the Center for Earth Observation Research, NPU. He had been a visiting scholar at Adelaide University, Adelaide, S.A., Australia, and Visiting Professor with Sydney University, Sydney, N.S.W., Australia, and Adelaide University. His research interests focuses on advanced machine vision and intelligent processing, including signal and image processing, computer vision, hyperspectral remote sensing, 3-D information acquisition and processing, neural network, and deep learning.

Dr. Mingyi was a recipient of 11 national and provincial scientific prizes and 2 teaching achievement prizes in China. He was the corecipient of the 2012 CVPR best paper award, the 2017 APSIPA ASC best deep/machine learning paper award, the 2017 DICTA best student paper award, etc. He was also a recipient of the government lifelong subsidy from the State Council of China in 1993 and 2017 Baosteel Outstanding Teacher Award. He has acted as a General Chair or TPC (Co)Chair, and Area Chair for a number of national and international conferences. He has been a member of the Advisory Committee of National Council for Higher Education on Electronics and Information in China, a member of Chinese Lunar Exploration Expert Group, the Vice-President of Shaanxi Institute of Electronics, and the Vice-Director of the Spectral Imaging Earth Observation Committee of China Committee of International Society of Digital Earth. He is an Associate Editor for the IEEE TRANSACTIONS ON GEOSCIENCE AND REMOTE SEnsing, a Guest Editor for the IEEE Journal of SELECTED Topics In APPLIED EARTh ObSERVATIONS AND REMOTE SENSing, and the Asia-Pacific Signal and Information Processing Theory and Methods (SIPTM) Association committee member. 


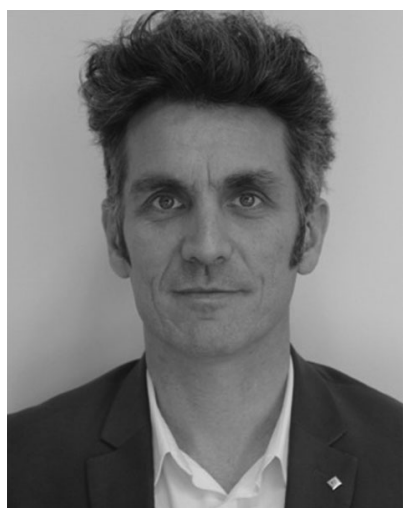

Jocelyn Chanussot (M'04-SM'04-F'12) received the M.Sc. degree in electrical engineering from the Grenoble Institute of Technology (Grenoble INP), Grenoble, France, in 1995, and the Ph.D. degree in electrical engineering from the Université de Savoie, Annecy, France, in 1998.

In 1999, he was with the Geography Imagery Perception Laboratory, Delegation Generale de l'Armement, French National Defense Department. Since 1999, he has been with Grenoble INP, where he is currently a Professor of signal and image processing. He is conducting his research at the Grenoble Images Speech Signals and Automatics Laboratory. He has been a visiting scholar at Stanford University, Stanford, CA, USA, KTH Royal Institute of Technology, Stockholm, Sweden, and National University of Singapore, Singapore. Since 2013, he has been an Adjunct Professor with the University of Iceland, Reykjavík, Iceland. During 2015-2017, he was a Visiting Professor with the University of California, Los Angeles, Los Angeles, CA, USA. His research interests include image analysis, multicomponent image processing, nonlinear filtering, and data fusion in remote sensing.

Dr. Chanussot is the founding President of the IEEE Geoscience and Remote Sensing French Chapter (2007-2010) who received the 2010 IEEE GRS-S Chapter Excellence Award. He was the corecipient of the NORSIG 2006 Best Student Paper Award, the IEEE GRSS 2011 and the 2015 Symposium Best Paper Award, the IEEE GRSS 2012 Transactions Prize Paper Award, and the IEEE GRSS 2013 Highest Impact Paper Award. He was a member of the IEEE Geoscience and Remote Sensing Society AdCom (2009-2010), and in charge of membership development. He was the General Chair of the first IEEE GRSS Workshop on Hyperspectral Image and Signal Processing, Evolution in Remote sensing (WHISPERS). He was the Chair (20092011) and Co-Chair of the GRS Data Fusion Technical Committee (2005-2008). He was a member of the Machine Learning for Signal Processing Technical Committee of the IEEE Signal Processing Society (2006-2008) and the Program Chair of the IEEE International Workshop on Machine Learning for Signal Processing, (2009). He was an Associate Editor for the IEEE Geoscience And Remote Sensing LetTers (2005-2007) and Pattern Recognition (2006-2008). Since 2007, he has been an Associate Editor for the IEEE TRAnSACTIONS On GEOSCIENCE AND REMOTE SENSING. He was the Editor-in-Chief of the IEEE Journal of Selected Topics in ApPlied EARTH ObSERVATIOnS AND ReMOTE SEnsing (2011-2015). In 2013, he was a Guest Editor for the Proceedings of the IEEE and, in 2014, a Guest Editor for the IEEE SIGNAL PROCESSING MAGAZINE. He is a member of the Institut Universitaire de France (2012-2017). 